\title{
COVID-19 pandemic and the social effects on clinic and scientific research: the "human factor"
}

\author{
Annalisa Cogliandro ${ }^{1,2}$ (I) $\cdot$ Mauro Barone ${ }^{1,2} \cdot$ Paolo Persichetti ${ }^{1,2}$
}

Received: 20 May 2020 / Accepted: 15 June 2020 / Published online: 24 June 2020

(C) Springer-Verlag GmbH Germany, part of Springer Nature 2020

The COVID-19 pandemic currently underway has overturned every personal, family, and work habit, generating new dynamics from the actual state of need whose consequences will be assessed over time. It is evident that remote work reduces distractions and maximizes the pro capite outcome of the single worker; however, the individual who spends his/her time in a stimulating environment, exchanging ideas and knowledge, increases not only his own creativity and inventiveness but even also those of the group he works with.

In the Anglo-Saxon culture, the term "water cooler talk" or "water cooler conversation" is what happens when colleagues take a break from work-related tasks and discuss their hobbies, interests, and personal issues. Great ideas have always arisen thanks to interpersonal exchanges. It is not possible to replace the disruptive effect of contact and physical interaction between researchers, teachers, and students as it happens in a university context. Emmanuel Kant underlined the way individuals are pushed towards society by different and opposite tendencies, he speaks of "unsociable sociability" [1] as one of the most powerful factors of historical development and progress, because it stimulates people to develop their skills. However, it must be governed by rules that ensure its functioning: only through the regulation given by "external laws" the antagonism of individuals can be realized in such a way everyone's freedom can coexist with the freedom of all the others.

In the medical-surgical field in general and especially in plastic surgery, the pandemic has generated the flowering of countless webinars sponsored by the national or international

Annalisa Cogliandro

a.cogliandro@unicampus.it

1 Plastic and Reconstructive Surgery Department, Campus Bio-Medico University of Rome, Via Alvaro del Portillo, 200 Rome, Italy

2 Research group "To be and to appear: Objective indication to Plastic Surgery” of Campus Bio-Medico University of Rome, Rome, Italy medical scientific societies when not organized by doctors considered points of reference for special topics as an alternative to the conferences that have necessarily been postponed due to COVID-19 pandemic effect.

These circumstances have allowed the state of the art updating but in the meantime, it has generated at least two critical issues whose impact will have to be followed over time [2]. The first critical issue is technical: the Internet guarantees immediate access to great number of information in an attempt to universalize knowledge, but who checks and certifies the correctness of the information given by the faculties of the webinar? Moreover, who is able to verify how these new pieces of information are learnt? What's the impact on personal surgical activity?

In fact, it is quite different to participate in the live conferences where close contact with tutors and mentors is provided, instead of listening to a webinar. The second important point is the so-called human-factor (this term is borrowed from the well-known growth factors in the biological field): medical knowledge has always benefited from the union of different thinking heads. In fact, many terms, entered into the daily practice as "brain storming" and "open space," generate the idea of a synergetic effect where inventiveness is enhanced through the physical contact, thanks to unexpected stimuli and contributions. Solo learning has been a fallback for this pandemic period but will never replace the role of the social impact on the knowledge progress.

The process of acquiring knowledge is not only the result of a sum of notions, but it grows through the relationships between the new concepts acquired and the experience completed and enriched by the interaction with other people.

"In life, a person can take one of two attitudes: to build or to plant. The builders might take years over their tasks, but one day, they finish what they're doing. Then they find that they're hemmed in by their own walls. Life loses its meaning when the building stops. Then there are those who plant. 
They endure storms and all the vicissitudes of the seasons, and they rarely rest. But unlike a building, a garden never stops growing. And while it requires the gardener's constant attention, it also allows life for the gardener to be a great adventure. Gardeners always recognize each other, because they know that in the history of each plant lies the growth of the whole World". [3] Paulo Coelho, from Brida.

\section{Compliance with ethical standards}

Conflict of interest All the authors declare that they have no conflict of interest.
Ethics approval This is not a scientific study. The Campus Bio Medico Ethics Committee has confirmed that no ethical approval is required.

Patient consent Not required. No patients involved in the manuscript.

\section{References}

1. Kant I (1784) "Idea for a Universal History with a Cosmopolitan Aim" (German: Idee zu einer allgemeinen Geschichte in weltbürgerlicher Absicht)

2. Mayer HF, Persichetti P (2020) Plastic surgery during the COVID19 pandemic times. Eur J Plast Surg 43:361-362

3. Coelho P (2009) "Brida" HarperCollins

Publisher's note Springer Nature remains neutral with regard to jurisdictional claims in published maps and institutional affiliations. 